\title{
USO DE ELEMENTOS DE VALOR PARA APOIAR A DEFINIÇÃO DE CONCEITOS DE PSS
}

Sânia da Costa Fernandes (saniafernandes@usp.br) - Departamento de Engenharia de Produção, Escola de Engenharia de São Carlos, Universidade de São Paulo.

Maiara Rosa (maiara.rosa@usp.br) - Departamento de Engenharia de Produção, Escola de Engenharia de São Carlos, Universidade de São Paulo.

Henrique Rozenfeld (roz@usp.br) - Departamento de Engenharia de Produção, Escola de Engenharia de São Carlos, Universidade de São Paulo.

\section{RESUMO}

Sistemas produto-serviço (PSS) são soluções que integram produtos e serviços de forma a suprir as necessidades de clientes por meio de maior valor percebido no uso. Para isso, as empresas devem enfatizar a criação da proposição de valor a fim de identificar os principais valores que a solução ofertada deve satisfazer. Este trabalho tem como objetivo propor uma ferramenta para a conceituação de um PSS durante a proposição de valor, associada a um conjunto de elementos de valor. Esta pesquisa adota a técnica de pesquisa-ação. A ferramenta foi verificada internamente e em duas aplicações baseadas em um estudo de caso realizado em uma empresa de manufatura de equipamentos para diagnóstico médico. Ela provê a identificação das necessidades, oportunidades e insights dos stakeholders, bem como de problemas que se deseja evitar ou solucionar. Esses resultados são utilizados como inspiração para a ideação de soluções e de suas características, juntamente com uma relação de possiveis elementos de valor que podem ser oferecidos em um PSS, sejam funcionais, emocionais, de mudança de vida elou sociais. Este trabalho contribui com as empresas de manufatura em processo de servitização para a criação de um PSS que enfoque nos valores elementares oferecidos aos stakeholders.

Palavras-chave: Sistemas produto-serviço; valor; ideação; conceitos; ferramenta

Área: Desenvolvimento de Sistemas Produto-Serviço (PSS)

\section{INTRODUÇÃO}

Empresas de manufatura são continuamente desafiadas pelo mercado a adicionar valor ao negócio e melhor atender as necessidades e desejos dos clientes. Uma solução que pode apoiar este desafio é o sistema produto-serviço (PSS - Product-Service System). Para o alcance do PSS, há a alteração do modelo tradicional de negócio, baseado na venda de produtos, para uma oferta integrada de produtos e serviços (BAINES et al., 2009; BOEHM; THOMAS, 2013; MARTINEZ et al., 2010; TAN; MCALOONE, 2006). Esse processo de transformação do modelo de negócio é denominado servitização (BAINES et al., 2009), que contempla a proposição de valor por meio da oferta de serviços para complementar aos produtos já comercializados.

Pieroni et al. (2016) apresentam o PSS Transition Framework, um guia para apoiar a transição para o modelo de negócio de PSS. Ele é constituído por nove fases, desde a análise do negócio até o lançamento da cadeia de valor. Uma das fases que influencia no sucesso do desenvolvimento e implementação do PSS é a proposição de valor. Ela objetiva compreender 
o perfil dos stakeholders (e não apenas dos clientes) para identificar o conjunto de benefícios que podem ser entregues por meio de uma solução (PIERONI et al., 2016). Os valores podem ser expressos a partir de atributos fundamentais, denominados por elementos de valor, que propiciam a criação de oportunidades (ALMQUIST et al., 2016) que fomentem a ideação de conceitos de PSS.

Dentre as ferramentas para a proposição de valor, destaca-se o Value Proposition Canvas $^{\circledR}$, o qual propõe identificar os principais problemas, os desejos e necessidades dos clientes a partir do alinhamento entre as soluções e os segmentos de clientes (OSTERWALDER et al., 2014). Entretanto, em aplicações do Value Proposition Canvas ${ }^{\circledR}$ verifica-se a necessidade de oportunidades de melhoria na ferramenta, as quais podem impactar na qualidade do resultado a ser obtido no processo de proposição de valor. Tal ferramenta instiga ao levantamento de informações redundantes, visto que as partes que a compõe se sobrepõem. Por exemplo, não há uma distinção clara quanto à categorização do que é uma tarefa dos clientes e os ganhos desejados pelos clientes. Além disso, as ideias geradas são provenientes puramente de um brainstorming, ou seja, não há um elo entre as etapas propostas na proposição de valor que direcione efetivamente à ideação.

A criação de uma ponte para o brainstorming é importante para garantir sua facilitação. As técnicas de brainstorming recomendadas na literatura, por exemplo, costumam ser acompanhadas de recomendações, como inserir restrições à ideação como forma de estimular novas ideias e evitar estagnação (PLATTNER, 2010). Assim, estabelecer elementos de valor para estimular a ideação do time pode auxiliar tanto a evitar estagnação quanto estimular a visualização de outras perspectivas em relação ao problema analisado.

O objetivo do presente trabalho é propor uma ferramenta para a proposição de valor de PSS no processo de servitização, associada a um conjunto de elementos de valor que fomentam a ideação de conceitos de PSS e que realizam a conexão entre o mapeamento do perfil dos stakeholders e a definição de propostas de valor.

O presente trabalho está estruturado como se segue. A seção 2 apresenta a explanação sobre os elementos de valor. A metodologia da pesquisa é descrita na seção 3. A seção 4 apresenta os resultados e a discussão em relação à ferramenta de proposição de valor e sua aplicação. Finalmente, a seção 5 delineia as conclusões da pesquisa.

\section{ELEMENTOS DE VALOR}

Almquist et al. (2016) identificaram trinta elementos de valor que podem ser relacionados às soluções oferecidas no mercado. Neste artigo, eles são utilizados como referência para a ideação de conceitos de PSS.

Os trinta elementos de valor são divididos em quatro categorias, as quais estendem a "Pirâmide de Necessidades" de Maslow (MASLOW, 1943) a partir do enfoque nas pessoas como clientes, considerando seus comportamentos em relação aos produtos e serviços. Com base no trabalho de Almquist et al. (2016), as seguintes definições são propostas para cada uma das categorias:

- Funcional: relaciona-se aos valores básicos para os stakeholders, sendo associados a finalidades práticas que refletem efetivamente em alguma função no dia-a-dia deles;

- Emocional: são os valores associados à geração de emoções positivas nos stakeholders;

- Mudança de vida: refere-se aos valores que se associam à capacidade de alterar ou influenciar a perspectiva sobre a qual os stakeholders analisam o mundo;

- Impacto social: são os valores relativos à busca do bem comum, com foco na melhoria 
da condição de vida das pessoas com as quais os stakeholders possuem empatia.

As categorias e seus respectivos elementos de valor são apresentados na Figura 1.

Figura 1. Elementos de valor e suas categorias. Fonte: Almquist et al. (2016)

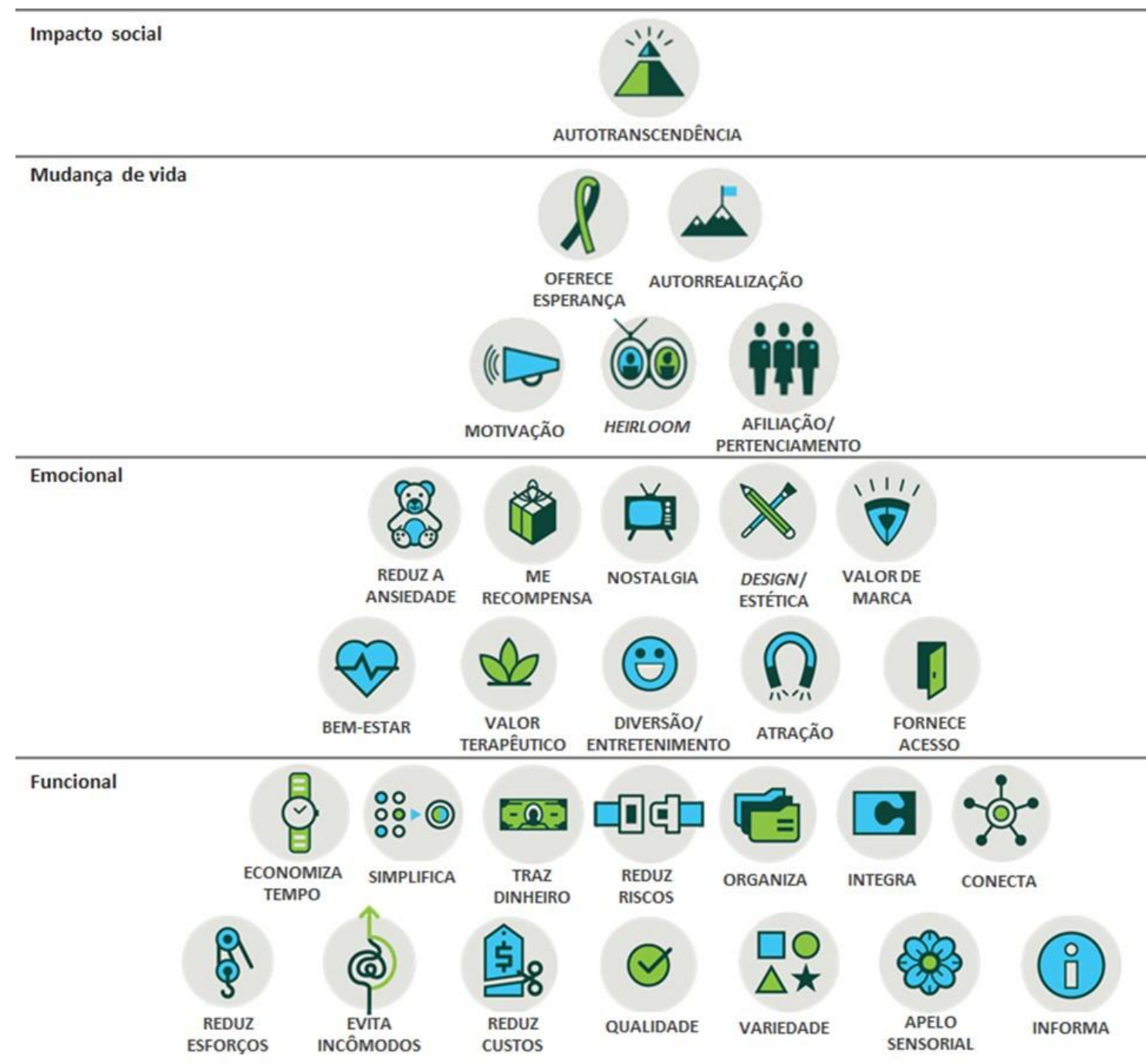

Ressalta-se que, segundo Almquist et al. (2016), uma única solução não é capaz de oferecer todos os elementos de valor. No entanto, soluções com um maior número de elementos de valor têm maior probabilidade de sucesso e aceitação do mercado.

\section{METODOLOGIA}

Esta pesquisa adota a técnica de pesquisa-ação para propor uma ferramenta de proposição de valor de PSS. A pesquisa-ação é um método científico que tem como foco o agir, apresentando caráter interativo de forma a buscar um completo entendimento de um sistema para a obtenção de soluções para um problema científico e organizacional em colaboração com a evolução da ciência (COUGHLAN; COGHLAN, 2009).

Neste trabalho foram aplicados dois ciclos de pesquisa-ação. Um ciclo de pesquisa-ação é composto pelos passos descritos abaixo, de acordo com Coughlan e Coghlan (2009), sendo precedidos pela identificação do contexto e do propósito da solução:

- Diagnóstico: identificação dos problemas que necessitam de solução e que serão o alvo 
da ação a ser tomada;

- Planejamento: envolve o planejamento da ação que será realizada de forma consistente aos problemas identificados;

- Implementação: é a efetivação da ação planejada em prol das mudanças desejadas;

- Avaliação: o fim do ciclo ocorre com a avaliação dos resultados da ação, de forma a realimentar o ciclo para a melhoria da solução.

A abordagem metodológica desta pesquisa foi a pesquisa-ação, a qual permite a utilização de outros métodos para apoiar a sua implementação. Neste trabalho, o método de estudo de caso foi utilizado como apoio à pesquisa-ação. Então, seguiu-se o processo descrito a seguir. No primeiro ciclo, a ferramenta para a proposição de valor no desenvolvimento de um PSS foi criada utilizando como referência as melhores práticas e informações identificadas em literatura. Ela também foi proposta como uma melhoria do Value Proposition Canvas ${ }^{\circledR}$ (OSTERWALDER et al., 2014) associada à inserção de um conjunto de elementos de valor (ALMQUIST et al., 2016) para serem utilizados como restrições para estímulo de ideação.

Ainda no primeiro ciclo da pesquisa-ação, a aplicabilidade inicial da ferramenta foi avaliada a partir de uma aplicação exemplar baseada em um estudo de caso prévio. Tal estudo de caso abrangeu o desenvolvimento de um PSS em uma empresa de manufatura de equipamentos para diagnóstico médico, e seguiu as seguintes etapas de acordo com o PSS Transition Framework: (i) análise prévia do negócio; (ii) proposição de valor com base em profundo entendimento dos stakeholders por meio da abordagem de design thinking; (iii) criação do um novo modelo de negócio; (iv) elaboração do business case, e (v) criação da arquitetura dos processos (PIERONI et al., 2016; ROSA et al., 2016). Uma aplicação da ferramenta, em caráter de teste, foi realizada com um especialista que foi consistentemente contextualizado em relação ao caso.

No segundo ciclo da pesquisa-ação, a ferramenta foi aprimorada de acordo com o feedback obtido no primeiro ciclo, e foi novamente aplicada. Essa aplicação ocorreu em um workshop com a participação de empresários e membros de conselhos de quinze empresas brasileiras. Ao final deste ciclo, houve novamente um processo de feedback, a partir do qual foram definidas outras melhorias para a ferramenta.

\section{RESULTADOS}

A ferramenta para a proposição de valor de PSS, bem como a explicação de seu funcionamento, é apresentada na seção 4.1. A segunda seção ilustra a aplicação da ferramenta com base no estudo de caso prévio realizado em uma empresa de manufatura de equipamentos para diagnóstico médico.

\subsection{Ferramenta de proposição de valor de PSS}

O Value Proposition Canvas ${ }^{\circledR}$ (OSTERWALDER et al., 2014), associado à inserção de elementos de valor (ALMQUIST et al., 2016), foi utilizado como inspiração para a criação da ferramenta de proposição de valor de PSS de modo que o entendimento do perfil dos stakeholders até a definição de propostas de soluções ocorresse de forma clara e concisa. A ferramenta, intitulada The Value Ring, é apresentada na Figura 2.

O Value Ring é composto por um quadro e um deck de cartas. O quadro contempla três partes principais: à esquerda, em forma de um rosto humano, encontra-se o perfil dos stakeholders; no diamante, na região central do quadro, são apresentados os elementos de valor que a proposição de valor pode satisfazer; à direita, em forma de um presente, é apresentada a 
proposta de valor em si, a qual compete às soluções de produtos, serviços e características de PSS a serem ofertadas. Ressalta-se que os elementos de valor foram dispostos em um diamante e não em uma pirâmide, como apresentado por Almquist et al. (2016). A utilização do diamante teve dois objetivos: (i) remeter à associação dos elementos ao valor, e (ii) evitar uma representação hierárquica dos elementos de valor, dado que a importância de um elemento de valor não pode ser previamente estabelecida e deve derivar do entendimento dos stakeholders.

Figura 2. The Value Ring. Fonte: Os autores.

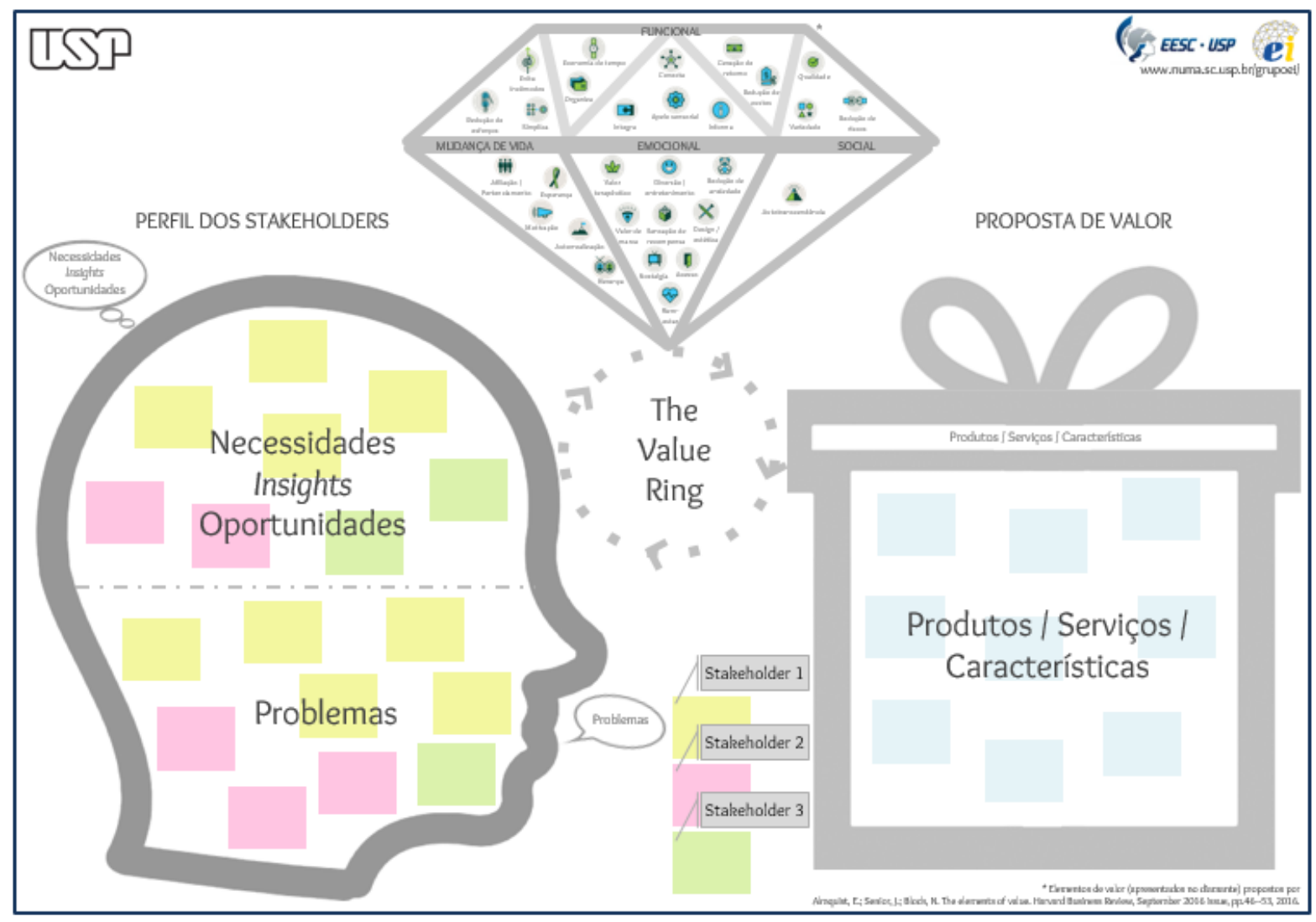

Cada carta do deck contém a definição e um exemplo de um dos elementos de valor, separadas por cores de acordo com a categoria ao qual pertencem.

No contexto do PSS Transition Framework (PIERONI et al., 2016), a ferramenta é aplicada na fase de proposição de valor, que ocorre após a realização da análise do negócio. Nessa fase, é realizado o mapeamento dos stakeholders para permitir o entendimento daqueles que possuem maior impacto para a proposição de valor. Sugere-se a realização de entrevistas e observação, sintetizando os dados levantados em mapas de empatia (PLATTNER, 2010) e mapas de jornada (LIEDTKA; OGILVIE, 2011). Portanto, o Value Ring é, inicialmente, associado a métodos selecionados para o entendimento dos stakeholders, provendo a identificação de suas necessidades, insights e oportunidades, que são descritas na parte superior do rosto humano. Também, são identificados os problemas que se deseja evitar ou solucionar, os quais são inseridos na parte de baixo do rosto. Recomenda-se a utilização de post-its de cores diferentes para cada stakeholder analisado de forma a garantir a distinção das informações, como ilustrado na Figura 2.

Em seguida, identifica-se a qual(is) elemento(s) de valor cada uma das necessidades, insights e oportunidades do perfil dos stakeholders está associada. Para facilitar tal identificação, é utilizado o deck de cartas que contém os elementos de valor. 
Com base nas informações obtidas no perfil dos stakeholders e no(s) respectivo(s) elemento(s) de valor aos quais estão relacionadas, estimula-se a proposição de diferentes soluções, incluindo produtos, serviços e características de PSS, que são dispostas na parte dedicada à proposta de valor. Também se recomenda a utilização de post-its para descrever as informações.

Ao final, após a definição de todas as soluções possíveis para oferecer os elementos de valor associados às necessidades, insights, oportunidades e problemas dos stakeholders analisados, identifica-se quais são os elementos de valor ainda não foram abordados. Considerando o objetivo da servitização, analisa-se, então, quais deles poderiam ser agregados às soluções de forma a satisfazer possíveis necessidades ou desejos não-explicitados pelos stakeholders.

As soluções mais pertinentes podem, então, ser combinadas em conceitos para serem testados pelos stakeholders e melhorados de acordo com o feedback obtido. Baseado no PSS Transition Framework (PIERONI et al., 2016), esse processo não é linear, e sim iterativo à medida que ocorre o progresso das demais fases.

A próxima seção apresenta a aplicação da ferramenta Value Ring em um caso de uma empresa de manufatura de equipamentos para diagnóstico médico.

\subsection{Aplicação do Value Ring}

A empresa na qual a ferramenta foi inicialmente aplicada possui um amplo portfólio de produtos, porém apenas o equipamento de diagnóstico por imagem foi alvo do processo de servitização. Usualmente, os produtos eram vendidos em transações $\mathrm{B} 2 \mathrm{~B}$ (business to business) com grandes clínicas, mas a empresa almejava ampliar sua participação no mercado a partir da oferta a consultórios. Para isto, o desenvolvimento de um PSS foi proposto com base no PSS Transition Framework (PIERONI et al., 2016).

Após mapeamento dos stakeholders, três deles foram considerados como mais impactantes e como principais alvos no processo de servitização: a própria empresa, os médicos e os pacientes finais. Foram realizadas entrevistas e observação, sintetizando os dados em mapas de empatia e mapas de jornada para identificar as principais necessidades, insights e oportunidades (referenciados pela letra "N") e os principais problemas (referenciados por "P" em relação ao que é possível de ser solucionado). Uma síntese das informações levantadas para cada stakeholder, bem como os elementos de valor aos quais elas estão relacionadas, é apresentada na Tabela 1.

A partir disto, foram propostos produtos, serviços e outras características da solução, que compõem a proposta de valor como um todo. Esses artefatos (S) que compuseram a solução no caso aplicado são, também, apresentados na Tabela 1, e foram associados aos seus respectivos problemas $(\mathrm{P})$ ou necessidades, insigths e oportunidades $(\mathrm{N})$, e aos elementos de valor que são por elas satisfeitos.

A fim de testar novamente a ferramenta, duas novas aplicações foram realizadas. Para isto, as necessidades, insights, oportunidades e problemas mais importantes identificados no estudo de caso inicial foram selecionados e explicitados na ferramenta. No primeiro teste, um especialista da área de Engenharia de Produção, com amplo conhecimento dos conceitos relacionados à proposição de valor, utilizou a ferramenta. Ele foi contextualizado de forma intensa com as informações identificadas nos perfis dos stakeholders e obteve êxito na criação de propostas de valor. A ferramenta cumpriu seu propósito, pois diferentes propostas de valor, com elevado número de elementos de valor, foram elencadas, atendendo a todas as necessidades e problemas identificados previamente. Após feedback, a ferramenta foi melhorada em alguns aspectos para aprimorar a sua usabilidade. 
Tabela 1. Solução proposta ao final do estudo de caso e a relação de suas partes com necessidades, insights, oportunidades e problemas, e com elementos de valor

\begin{tabular}{|c|c|c|c|c|c|c|c|c|c|c|c|c|c|c|c|c|c|c|c|c|c|c|c|c|c|c|}
\hline \multirow{12}{*}{ 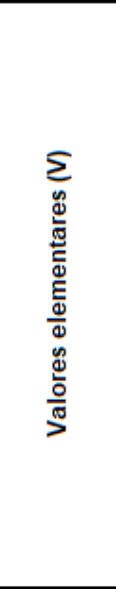 } & $\sum^{2}$ & Afiliação/Pertencimento & & & & & & & & & & & & & & & & & & & $\times$ & & & $\times$ & & \\
\hline & $\sum_{1}^{-}$ & Acesso & & & & & & & & $\times$ & & & & & & $\times$ & & & & & & $\times$ & & & & \\
\hline & 은 & Redução de ansiedade & & & & & & & $\times$ & & $\times$ & $\times$ & $\times$ & $\times$ & $\times$ & & & & & & & & & & $\times$ & \\
\hline & $\stackrel{9}{9}$ & Conecta & & & & & & & & & & & & $\times$ & $\times$ & & & & & & & & & $\times$ & & \\
\hline & $\stackrel{\infty}{9}$ & Integra & & $\times$ & $\times$ & $\times$ & $\times$ & & & & & & & & & & & & & & & & & & & \\
\hline & $\hat{9}$ & Economia de tempo & $\times$ & $\times$ & $\times$ & & $\times$ & $\times$ & $\times$ & & $\times$ & $\times$ & $\times$ & $\times$ & $\times$ & & $\times$ & $\times$ & $\times$ & $\times$ & & & & & $\times$ & $\times$ \\
\hline & $\stackrel{8}{\circ}$ & Redução de custos & $\times$ & $\times$ & $\times$ & & $\times$ & $\times$ & $\times$ & & $\times$ & $\times$ & $\times$ & & & $\times$ & $\times$ & & & & & & & & & \\
\hline & $\stackrel{5}{3}$ & Informa & & & $\times$ & & & & & & & & & $\times$ & $\times$ & & $\times$ & $\times$ & $\times$ & $\times$ & & $\times$ & $\times$ & & $\times$ & \\
\hline & \$ & Geração de receita (médico) & & & & & & & & $\times$ & & & & & & & & & & & & & $\times$ & & & $\times$ \\
\hline & 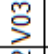 & Redução de risco & & & $\times$ & $\times$ & $\times$ & $\times$ & & & $\times$ & $\times$ & & & $\times$ & & $\times$ & & & & & & & & & \\
\hline & N & Evita incômodo & & $\times$ & & & $\times$ & $\times$ & & & $\times$ & $\times$ & & & $\times$ & & & & & & & & & & & \\
\hline & 5 & Qualidade & $\times$ & $\times$ & $\times$ & & $\times$ & $\times$ & $\times$ & & $\times$ & $\times$ & $\times$ & $\times$ & & $\times$ & $\times$ & $\times$ & $\times$ & $\times$ & & $\times$ & & & $\times$ & \\
\hline \multirow{3}{*}{ 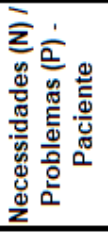 } & 옴 & $\begin{array}{c}\text { Alto custo com deslocamento ao } \\
\text { consultório, esperas e ausência no } \\
\text { trabalho }\end{array}$ & & & & & & & & & & & & $\times$ & & & & & & & & & & & & $\times$ \\
\hline & $\stackrel{2}{\circ}$ & $\begin{array}{c}\text { Obter a resposta do laudo no } \\
\text { momento em que está no consultório }\end{array}$ & $\times$ & & & & & & & & & & & & & & & & & & & & & & $\times$ & \\
\hline & 을 & Rápido atendimento & $\times$ & & & & & & & & & & & & & & & & & & & & & & & \\
\hline \multirow{5}{*}{ 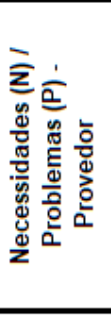 } & $\stackrel{2}{\circ}$ & $\begin{array}{l}\text { Muitas reclamações devido a quebras } \\
\text { e descalibração }\end{array}$ & $\times$ & & $\times$ & & $\times$ & $\times$ & $\times$ & & $\times$ & $\times$ & $\times$ & $\times$ & $\times$ & $\times$ & $\times$ & $\times$ & $\times$ & $\times$ & & & & & & \\
\hline & $\hat{\circ}$ & $\begin{array}{l}\text { Necessidade de muita mão-de-obra } \\
\text { disponivel para manutenção }\end{array}$ & $\times$ & & & & & & & & & $\times$ & & & $\times$ & & $\times$ & $\times$ & $\times$ & $\times$ & & & & & & \\
\hline & $\begin{array}{l}\square \\
\square\end{array}$ & Elevada inadimplência & & & & $\times$ & & & & $\times$ & & & & & & & & & & & & & & & & \\
\hline & $\stackrel{0}{\circ}$ & Faturamento recorrente & & & & & & & & $\times$ & & & & & & & & & & & & & & & & \\
\hline & $\stackrel{2}{0}$ & Aumento de market share & & & & & & & & $\times$ & & & & & & & & & & & & & & & & \\
\hline \multirow{9}{*}{ 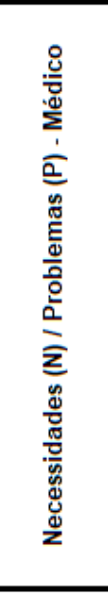 } & 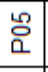 & $\begin{array}{l}\text { Não tem dinheiro para comprar o } \\
\text { equipamento }\end{array}$ & & & & & & & & $\times$ & & & & & & & & & & & & & & & & \\
\hline & ¿ & $\begin{array}{l}\text { Equipamento descalibra } \\
\text { constantemente }\end{array}$ & $\times$ & & & & $\times$ & $\times$ & $\times$ & & & $\times$ & & & & & $\times$ & $\times$ & $\times$ & & & & & & & \\
\hline & ஜ. & Operador não possui domínio técnico & & & & & & & & & & & & & & & $\times$ & $\times$ & $\times$ & $\times$ & $\times$ & & & & & \\
\hline & ণั & $\begin{array}{l}\text { Ocorrência de muitas paradas do } \\
\text { equipamento por falhas }\end{array}$ & $\times$ & $\times$ & $\times$ & & $\times$ & $\times$ & $\times$ & & $\times$ & $\times$ & $\times$ & $\times$ & $x$ & $\times$ & $\times$ & $\times$ & & & & & & & & \\
\hline & $\overline{0}$ & Alto custo de manutenção & $\times$ & $\times$ & $\times$ & & $\times$ & $\times$ & $\times$ & & $\times$ & $\times$ & $\times$ & & $\times$ & $\times$ & & & & & & & & & & \\
\hline & ¿ & Fonte adicional de faturamento & $\times$ & & & & & & & & & & & & & & & & & & & & $\times$ & & & \\
\hline & ஜ & $\begin{array}{l}\text { Equipamento disponivel } 24 / 7 \text { ( } 24 \\
\text { horas, por } 7 \text { dias) sem quebrar }\end{array}$ & $\times$ & $\times$ & $\times$ & & $\times$ & $\times$ & $\times$ & & $\times$ & $\times$ & $\times$ & $\times$ & & $\times$ & $\times$ & $\times$ & $\times$ & $\times$ & & & & & & \\
\hline & ণิ & Mostrar modernidade & $\times$ & & & & & & & & & & & & & & & & & & & & & & & \\
\hline & 은 & $\begin{array}{c}\text { Fazer imagem na hora, no próprio } \\
\text { consultório }\end{array}$ & $\times$ & & & & & & & & & & & & & & & & & & & & & & & \\
\hline & & \multirow[t]{2}{*}{ 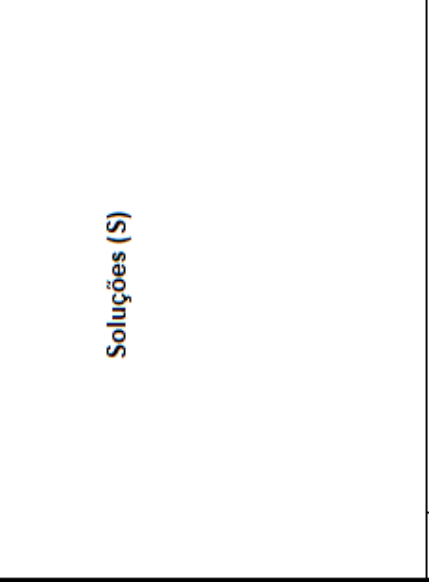 } & 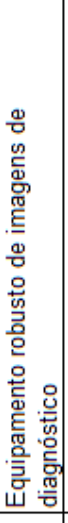 & 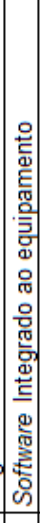 & 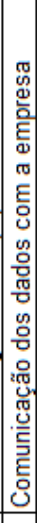 & 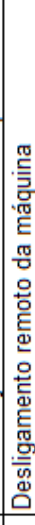 & 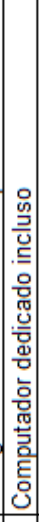 & 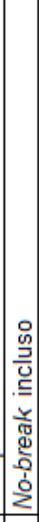 & 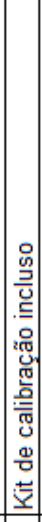 & 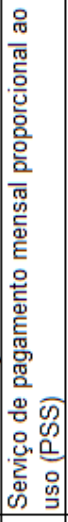 & 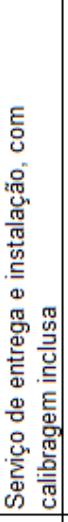 & 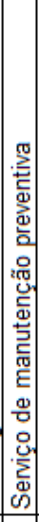 & 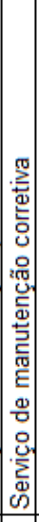 & 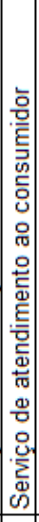 & 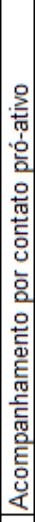 & 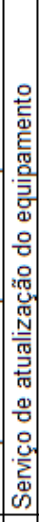 & 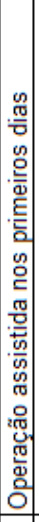 & 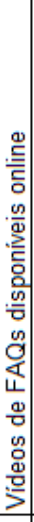 & 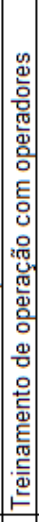 & 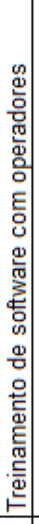 & 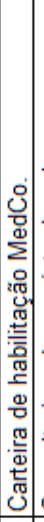 & 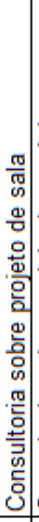 & 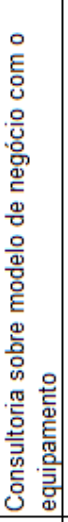 & 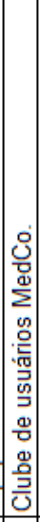 & 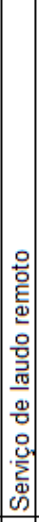 & 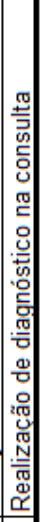 \\
\hline & & & চ & ํํㅇ & 竞 & 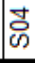 & $\begin{array}{l}0 \\
0 \\
0\end{array}$ & 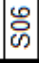 & 闹 & 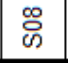 & 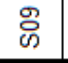 & 은 & $\bar{C}$ & $\frac{N}{\infty}$ & $\frac{m}{n}$ & $\frac{ \pm}{6}$ & $\frac{5}{6}$ & $\frac{6}{n}$ & हn & $\frac{\infty}{\infty}$ & $\frac{9}{\infty}$ & స్ & สี & ふิ & స్ & సี \\
\hline
\end{tabular}


Um segundo teste foi realizado por meio de um workshop com um grupo de quinze empresários e membros de conselhos de empresas de diferentes setores de negócio, compreendendo desde commodities até serviços bancários. Esse grupo foi brevemente contextualizado com os conceitos de proposição de valor e com as informações pertinentes ao estudo de caso da empresa de manufatura de equipamento de diagnóstico médico.

Nesse segundo teste não foram observados problemas em relação ao uso da ferramenta. No entanto, constataram-se dificuldades em relação ao entendimento dos participantes de que a proposição de valor deve ocorrer anteriormente à análise de questões financeiras (que remetem ao business case). A cada sugestão de solução, os participantes ficavam estagnados à tentativa de calcular a viabilidade econômica da proposta. Assim, apesar da facilidade em utilizar a ferramenta, houve uma dificuldade em aceitar a premissa de que o momento para se realizar as análises de viabilidade é após a primeira proposição e combinação das soluções e que o processo é iterativo. Além do mais, o tempo pré-determinado para o workshop (cerca de vinte minutos) não foi suficiente. Apenas cerca de dois a três elementos de valor puderam ser analisados.

As dificuldades constatadas podem indicar dois vieses. Pode ser necessário estabelecer uma contextualização mais clara a fim de se garantir o entendimento dos participantes de que a proposição de valor deve, inicialmente, abranger o maior número possível de propostas de soluções no processo de ideação, alterando o mindset dos participantes para ignorar, momentaneamente, a questão de viabilidade econômica. As dificuldades também podem representar uma falta de entendimento mais aprofundado do problema, conduzindo à conclusão de que a participação integral do time em todas as etapas da proposição de valor no processo de servitização é fundamental. Ambas as constatações são compatíveis com as conclusões que já haviam derivado do estudo de caso original, as quais indicavam como maior problema a não-adoção de um mindset adequado pelo time para evitar fixação de ideias e estimular um esforço centrado em valores humanos dos stakeholders (ROSA et al., 2016).

\section{CONCLUSÃO}

O Value Ring demonstrou-se como uma ferramenta útil para a ideação de soluções no processo de servitização, auxiliando na expansão dos conceitos e do oferecimento de maior valor percebido no uso. A inserção de elementos de valor capazes de restringir o brainstorming e estimular a criação de novas ideias se mostrou válido e atingiu o objetivo proposto: fomentar a proposição de valor na conceituação de um PSS. Ressalta-se, todavia, que melhorias serão propostas para a ferramenta a partir de novos ciclos de pesquisa-ação.

Com o objetivo de melhor entender e solucionar as dificuldades observadas durante a aplicação da ferramenta, como a excessiva ênfase na análise de viabilidade econômica pelos participantes, a ferramenta deverá ser utilizada em diferentes situações: considerando ou não o contexto do framework de servitização - PSS Transition Framework (PIERONI et al., 2016).

Apesar da aplicabilidade da ferramenta para o propósito ao qual se propõe, nota-se a necessidade de uma melhor preparação dos participantes sobre os conceitos e a importância da proposição de valor. Outro aspecto importante é a duração das atividades com a ferramenta. Em dinâmicas de curta duração (cerca de vinte minutos), é possível avaliar de dois a três elementos de valor, somente. É preciso estimular, entre os participantes, uma discussão focada, de modo que o tempo de aplicação não seja excessivamente longo.

Em relação ao comportamento dos empresários e membros de conselho durante a dinâmica, surgem algumas hipóteses. Eles podem estar acostumados não a gerar, mas a avaliar e melhorar propostas de valor com base em informações e estudos preliminares. Deve-se, então, 
analisar a importância de uma possível participação de pessoas de outros níveis hierárquicos no processo de ideação, bem como na avaliação das propostas de soluções obtidas a partir de informações melhor fundamentadas sobre as soluções (como o business case).

No entanto, o workshop foi útil para a sensibilização dos empresários e membros de conselho quanto à importância da servitização, do entendimento do perfil dos stakeholders nesse processo e da proposição de valor para se elencar soluções que maximizem o valor do negócio.

Como passos futuros para esta pesquisa, propõe-se a realização de novos ciclos de pesquisaação para aprimorar a ferramenta de forma evolutiva e garantir a efetividade de sua aplicabilidade.

\section{REFERÊNCIAS}

ALMQUIST, E.; SENIOR, J.; BLOCH, N. The elements of value. Harvard Business Review, n. September, p. 46-53, 2016.

BAINES, T. et al. The servitization of manufacturing: a review of literature and reflection on future challenges. Journal of Manufacturing Technology Management, v. 20, n. 5, p. 547-567, 2009.

BOEHM, M.; THOMAS, O. Looking beyond the rim of one's teacup: A multidisciplinary literature review of Product-Service Systems in Information Systems, Business Management, and Engineering \& Design. Journal of Cleaner Production, v. 51, p. 245-250, 2013.

COUGHLAN, P.; COGHLAN, D. Action Research. Researching Operations Management, 2009.

LIEDTKA, J.; OGILVIE, T. Designing for growth: a design thinking tool kit for managers, Columbia University Press, USA, 2011.

MARTINEZ, V. et al. Challenges in transforming manufacturing organisations into productservice providers. Journal of Manufacturing Technology Management, v. 21, n. 4, p. 449-469, 2010.

MASLOW, A. H. A theory of human motivation. Psychological Review, v. 50, n. 4, p. 370396, 1943.

OSTERWALDER, A. et al. Value Proposition Design. Wiley, 2014, 320 p.

PIERONI, M. et al. Transforming a Traditional Product Offer into PSS: A Practical Application. Procedia CIRP, v. 47, p. 412-417, 2016.

PLATTNER, H. Bootcamp Bootleg. Palo Auto: Design School Stanford, 2010, 47 p.

ROSA, M. et al. Application of design thinking towards a PSS concept definition: a case study. International Conference on Transdisciplinary Engineering. Anais...IOS Press, 2016.

TAN, A. R.; MCALOONE, T. C. Characteristics of strategies in product/service-system development. (D. Marjanovic, Ed.) International Conference on Design. Anais...Dubrovnik, Croatia: Faculty of Mechanical Engineering and Naval Architecture, 2006. 


\section{AGRADECIMENTOS}

Este projeto é apoiado pelo processo $n^{0}$ 2015/23094-6, Fundação de Amparo à Pesquisa do Estado de São Paulo (FAPESP). Os autores agradecem o apoio da FAPESP e da Coordenação de Aperfeiçoamento de Pessoal de Nível Superior (CAPES). 